\title{
Is single-molecule fluorescence spectroscopy ready to join the organic chemistry toolkit? A test case involving click chemistry
}

\author{
Juan C. Scaiano* and Anabel E. Lanterna \\ Department of Chemistry and Biomolecular Sciences and Centre for Catalysis Research and Innovation (CCRI), University \\ of Ottawa. 10 Marie Curie, Ottawa, ON K1N 6N5, Canada
}

Corresponding author's e-mail: titoscaiano@mac.com

\begin{abstract}
Single Molecule Spectroscopy (SMS) has matured to a point where it can be used as a convenient tool to guide organic synthesis and drug discovery, particularly applicable to catalytic systems where questions related to homogeneous $v s$. heterogeneous pathways are important. SMS can look at intimate mechanistic details that can inspire major improvements of the catalyst performance, its recovery and reuse. Here we use the click reaction between alkynes and azides as an example where improvements at the bench have been inspired and validated using single-molecule fluorescence spectroscopy.
\end{abstract}

\section{INTRODUCTION}

The last decade has seen major advancements in the tools for single molecule spectroscopy (SMS), largely due to improvements in microscopy, light sources, super-stable fluorescent dyes and an increased understanding of the physical principles that today enable spatial resolution much better than that allowed by the diffraction limit. ${ }^{1}$ These advancements have enabled admirable progress in biology, including in vivo detection of intracellular processes with exquisite spatial and temporal resolution. ${ }^{2,3}$ The progress in the utilization of advanced microscopy in chemistry and catalysis has been significantly slower, but a number of examples have been reported ${ }^{4-8}$ in which SMS has enabled the understanding of some reaction mechanisms at the single molecule level, and in particular, in the case of catalysis with single catalytic site resolution. Work on catalytic reactions on solid particles by Chen, ${ }^{9-13}$ Hofkens/Roeffaers, ${ }^{14-17}$ Majima, ${ }^{18}$ Goldsmith ${ }^{19}$ and others, ${ }^{20,21}$ sets the stage to transfer this new knowledge back to the organic laboratory. Inspired by these contributions and by our own experience and interests we embarked in a project to study a reaction at the bench, characterize it at the single molecule-single catalytic site level, use the knowledge acquired to improve the catalytic process at the bench level and finally, return to single molecule microscopy studies to verify/validate the improvements observed in the lab. This contribution tells the story of a successful journey to improve a click reaction; while it centers on our own work, we acknowledge that the contributions from other laboratories have been both, enabling and inspiring.

Many reactions could have been selected for our catalysis journey; our choice of click chemistry followed the observation that for the commercial copper-on-charcoal (Cu-oncharcoal) catalyst only $0.003 \%$ of the surface was active, in spite of its good catalytic performance in organic synthesis. ${ }^{22}$ It seemed that starting with low surface efficiency would pro- vide a good test case for the idea that single-molecule fluorescence spectroscopy is ready to join the organic chemistry toolkit.

In the past numerous techniques developed for physics or physical chemistry made their way to mainstream organic chemistry as tools to understand reactions mechanisms and kinetics. With this knowledge in hand it is possible to improve synthetic procedures making them "greener", increase selectivity, reduce reaction times, energy and solvents required, and in the case of catalysis increase recovery and reuse of catalysts. Catalyst testing frequently involves reliance on a trialand-error approach. This is where high-throughput screening laid its roots, being recognized as a powerful tool. ${ }^{23}$ However, as much as this technique may speed up the discovery process, it essentially relies on chance. The fact is that trial-and-error, even if accelerated, provides little or no mechanistic information. A new way to change the paradigm of organic synthesis involves using advanced single-molecule strategies as a routine tool to improve chemical manufacturing. ${ }^{14,16,21,24}$ The detailed knowledge of how and for how long a single molecule of reagent or product may interact with a catalyst, can be translated into improvements to the reaction in the laboratory and, eventually, at the industrial level.

Single molecule techniques have reached a point in which they are amenable to studies in organic synthesis and catalysis; $;^{9,11,12,25}$ they have developed to a point where organic chemistry practitioners can take full advantage of them to improve organic reactions at the bench and, eventually, at the manufacturing level. To illustrate our proposal, we use as an example the copper catalyzed click reaction between alkynes and azides, in which the copper is contained in a variety of heterogeneous systems. We show how this catalytic process was improved by over a factor of ten, entirely inspired by observations based on SMS; further, these ideas were used to develop a new copper-based photoactivated click catalyst. ${ }^{26}$ 
The ideas discussed here are part of a strategy implemented in our group to which we refer as "from the mole to the molecule", implying that the knowledge at the bench level can be taken and used to develop the reagents and methodologies to examine the reaction at the single molecule level. Arguably far more difficult, but with better practical prognosis is the reverse statement, "from the molecule to the mole", where we take the knowledge acquired at the molecular level, for example using SMS, to improve bench and manufacturing processes (Chart 1); an approach facilitated by studies by Blum, ${ }^{4,5}$ Hofkens, ${ }^{16}$ $\mathrm{Chen}^{9}$ and others ${ }^{14,17,21}$ in different systems. It is this ambitious concept that we believe will be attractive in areas of organic synthesis and in particular drug discovery.

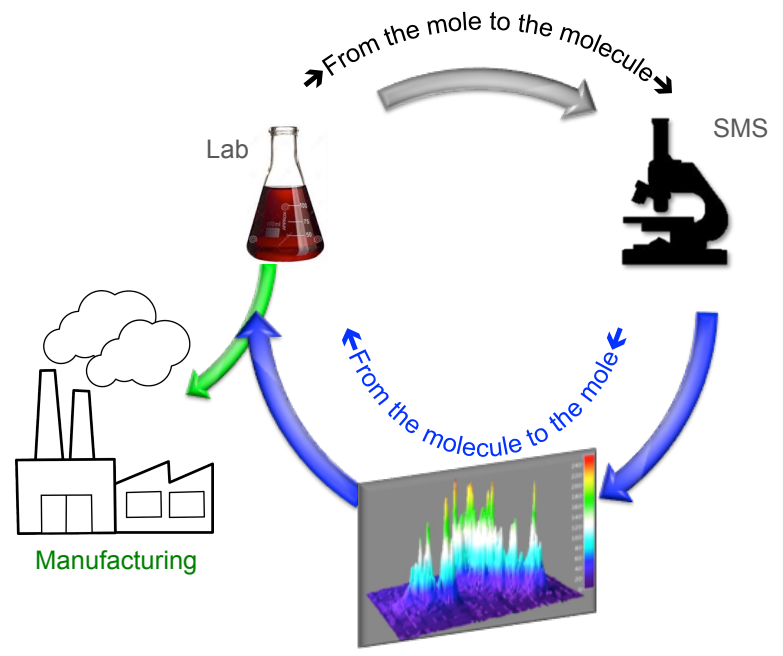

Chart 1. Flow chart showing the strategy approach used for singlemolecule studies in catalysis.

\section{THE CLICK REACTION}

The catalytic "click" chemistry was introduced by Sharpless et al. in $2001,{ }^{27}$ referring to the generation of new chemical units by selective heteroatom links $(\mathrm{C}-\mathrm{X}-\mathrm{C})$. According to them, a desirable click chemistry reaction must be modular, wide in scope, give very high yields, and be stereospecific. The required process characteristics include simple reaction conditions, readily available starting materials and reagents, the use of no solvent or a solvent that is benign or easily removed, and simple product isolation. The best known reaction that fits most of these conditions is the formation of 1,2,3-triazole by copper(I)-catalyzed cyclization between azides and terminal alkynes (CuAAC) as shown in Scheme 1.28,29

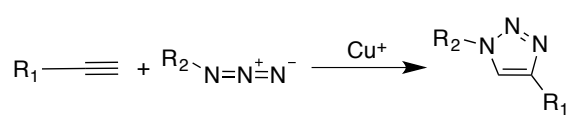

Scheme 1. Formation of 1,2,3-triazole by copper(I)-catalysed cycloaddition between azides and terminal alkynes (CuAAC).

There are many suggested mechanisms for the click reaction although most of them coincide with a pre-association step in which the alkyne moiety can interact with the catalyst forming what many have called a $\mathrm{Cu}$-acetylide complex (Scheme 2). ${ }^{29}$ ${ }^{32}$ This pre-association can facilitate single molecule studies at sub-nanomolar concentrations and help demonstrate catalysis occurring in the heterogeneous phase on the superficial catalytic sites (vide infra).

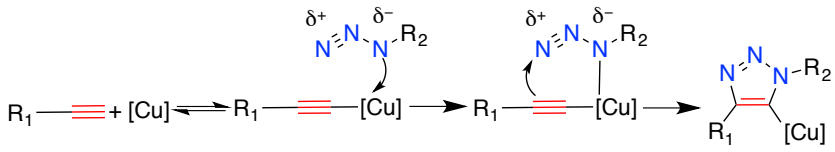

Scheme 2. Simplified proposed mechanism for the formation of 1,2,3triazole ring during $\mathrm{CuAAC}$. $[\mathrm{Cu}]$ denotes either a single-metal center $\mathrm{CuL}_{\mathrm{x}}$ or a di-/oligonuclear cluster $\mathrm{Cu}_{\mathrm{x}} \mathrm{L}_{\mathrm{y}} .{ }^{32}$

The CuAAC has been extensively studied using soluble copper species, although in the last few years there are some examples in the literature that show efforts pursuing the use of copper-based heterogeneous catalysts. ${ }^{33-35}$ The use of copper nanoparticles ${ }^{36,37}$ was one of the first steps towards the use of heterogeneous catalysts for $\mathrm{CuAAC}$ due to the potential enhancement of the catalytic activity by nanostructured metals. The well-known instability of colloidal CuNPs,${ }^{37}$ was overcome by replacing them with supported copper nanostructures. ${ }^{38}$ The use of $\mathrm{Cu}$-on-charcoal was first described by Lipshutz et al in $2006^{39}$ for it use in click chemistry. Since then many efforts have been done to develop multicomponent systems based on $\mathrm{Cu}$-on-charcoal, ${ }^{36,38,40,41}$ including the use of conventional bench scale techniques in order to determine whether or not the catalysis occurs in the heterogeneous phase.

The question of whether or not a reaction occurs in heterogeneous or homogeneous phase is crucial in catalysis. ${ }^{43-45}$ It is important to recognize that many processes cannot be unequivocally identified using this conventional classification (Chart 2), ${ }^{45}$ but rather, with an intermediate situation where different conditions may prevail: (C) active catalytic species can leach and diffuse away the surface of an heterogeneous material to react in the homogenous phase (homogeneous catalysis), ${ }^{46}$ (D) an homogeneous material can generate in situ heterogeneous species that are the truly catalytic active species (heterogeneous catalysis), ${ }^{47}$ or, (E) the heterogeneous catalyst releases the active catalytic species that after catalysis are redeposited on the catalyst surface (homogeneous catalysis/heterogeneous catalyst), ${ }^{44,48}$ Chart 2 . The catalytic site, "C", can be a monomeric species, part of a nanostructure composed of a single material, or part of a hybrid structure, frequently including a support material. While many attempts to address these concerns have been reported in the past, they generally involve regular bench scale strategies. Thus, detection of nanoparticulates by electronic microscopy, quantification of leached species by analytical techniques, chemical poisoning, among others. ${ }^{44}$ Nevertheless, if the catalytic mechanism involves the last-described situation (i.e., 'E'), bench scale strategies become more challenged as they can only detect catalyst leaching if it accumulates for a considerable period of time, and negative results cannot exclude this mechanism in Chart $2 .{ }^{49}$ Therefore, the interaction between a single molecule and a single catalytic site cannot be monitored, and sometimes more sophisticated in situ, real time techniques are needed to establish which mechanism is taking place.$^{50-52}$ Establishing heterogeneous catalysis does not necessarily rule out the occurrence of homogeneous catalysis; testing for homogeneous contributions using SMS can be challenging. In a study of click chemistry catalyzed by colloidal copper we were able to establish that at most $5 \%$ of the reaction involved homogeneous processes (vide infra)..$^{53}$ 
Conventional catalysis classification

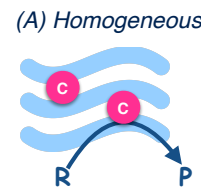

(B) Heterogeneous

\section{Exceptions to conventional catalysis classification}
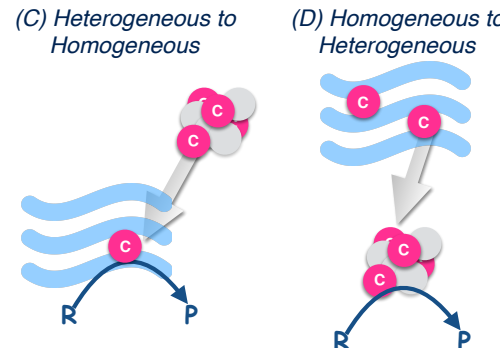

(E) Heterogeneous to Homogeneous \& back

Chart 2. Plausible processes that take place during catalysis. Conventional homogeneous and heterogeneous catalysis are represented by (A) and (B). The gray circles in (B) illustrate the possible involvement of supports, active or not, that are frequently part of heterogeneous catalysts. In (C) the active catalytic species can leach and diffuse away the surface of an heterogeneous material to react in the homogenous phase; in (D) a homogeneous catalyst forms complex insoluble nanostructures that act as in situ heterogeneous catalysts, and in (E) the heterogeneous catalyst releases the active catalytic species that after catalysis are re-deposited on the catalyst surface.

\section{THE TOOLS: TOTAL INTERNAL REFLECTION FLUORESCENCE MICROSCOPY}

Total Internal Reflection Fluorescence Microscopy (TIRFM) is a powerful tool that enables detection of fluorescent probes in a very narrow sub-micrometer specimen region. The principle of the technique is based on the interaction of an electromagnetic field (light) with a transparent surface (i.e., glass). Changes in the refractive index between the different surfaces deflect the light beam; thus, depending on the incident angle and the refractive index of the two surfaces (i.e. usually the contact area between a specimen and a glass coverslip), the light can be refracted or reflected. When the incident angle is appropriate (>critical angle) $100 \%$ of the light can be reflected, which is known as "total internal reflection". This can only happen in situations in which the light encounters a boundary to a medium with a lower refractive index. Although the light cannot penetrate the second medium, this light-matter interaction generates an electromagnetic field, called evanescent wave, adjacent to the interface. The evanescent wave has the same frequency as the incident light and its intensity decreases exponentially with the distance from the surface (normal direction to the interface), thus this field extends only a few hundreds nanometers into the specimen (Chart 3). Therefore, only the fluorophores placed within $\sim 100 \mathrm{~nm}$ or closer to the glass surface are submitted to the excitation light and can be "observed" by TIRFM. ${ }^{54}$

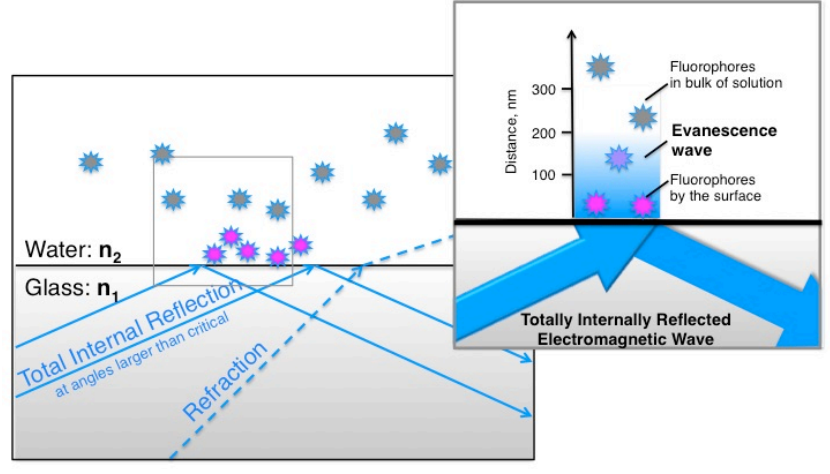

Chart 3. The light rays incident on the boundary between two transparent materials (water $\left(\mathrm{n}_{2}\right)$ and glass $\left(\mathrm{n}_{1}\right) ; \mathrm{n}_{1}>\mathrm{n}_{2}$ ) with an angle greater or equal to the critical angle are $100 \%$ reflected, a process known as Total Internal Reflection (TIR). The concomitant electromagnetic field induced in the second medium (water) is called evanescence wave. Its intensity decays exponentially from the interface surface exciting only the fluorophores molecules in close proximity.

When using TIRFM, the CuAAC reaction is monitored by flowing the reagents through a flow cell reactor that contains the supported catalyst. The resulting emission is recorded and can be observed as bright bursting events on a dark background. The analysis of bursts at localized spots yields fluorescence intensity versus time trajectories (vide infra). Single molecule events are confirmed by the rise and fall of the emission intensity, in contrast to the step-wise change in "on" intensity within a trajectory when monitoring multiple dyes ${ }^{55}$ (see Chart 4). Sudden intensity drops account for product desorption from the catalyst, or product degradation. The latter should decrease at lower laser power and this can relate to the duration of the "ON-time" periods in the intensity traces. When the affinity of the product for the catalytic site is weak, the migration of the product out of the evanescence field region is possible within the time scale of the experiment (frequently around $100 \mathrm{~s}$ ).

Single-molecule analysis requires a "significant" signal change in order to detect molecular changes. Namely, OFFON blinking or emission red-shift are the desired switching behavior on single molecules. ${ }^{6}$ The next section deals with the reasoning behind the selection of the fluorophores.
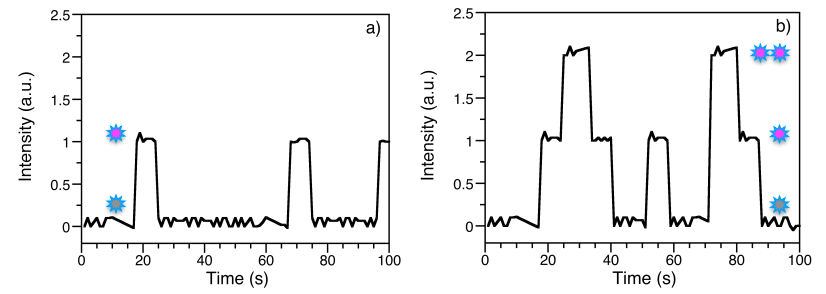

Chart 4. Expected idealized intensity trajectories for a) single-molecule events and b) multiple-molecules events occurring at one catalytic site (heterogeneous catalysis)

\section{Selecting fluorophores}

The chemical reactions that can be studied with singlemolecule microscopy based on fluorescence spectroscopy can be classified in two categories: ${ }^{6}$ Blum classifies the reactions as those that occur with structural changes on the reactant molecule giving rise to products with different emission properties (participant dye) ${ }^{56}$ and those that require a dye tagged to the reactant molecules to sense the progress of the reaction. 
The second category involves reactants that have attached a dye in order to follow their reaction, usually called spectator dye. Although the first approach is easier to follow by spectroscopic changes, it usually requires a laborious design of the probe molecule to suit the chemical requirements for the studied system. The second approach requires the careful selection of dyes, although this is becoming simpler as the increased use of single molecule spectroscopy in bioimaging has attracted the attention of manufacturers. This second approach is also advantageous in that it requires less modification of the reaction of interest. Nowadays, the library of dyes with useful functional groups is growing fast as more robust dyes are needed and many have become commercially available..$^{57,58}$

The CuAAC belongs to the second category described above. Due to the absence of emissive properties, not only the alkyne but also the azide need to be tethered to a dye. In our studies the selection of the probe molecules was done based on a wellknown energy transfer mechanism between two light-sensitive molecules, the Förster resonance energy transfer (FRET).$^{59,60}$ This mechanism involves a non-emissive energy transfer through long-range dipole-dipole interactions from the donor chromophore to the acceptor chromophore. Because the strength of the interaction is inversely proportional to the sixth power of the distance between the two molecules, the energy transfer can only occur when they are in close proximity (typically 1 to $10 \mathrm{~nm}$ ), and therefore the technique is very sensitive to the changes in distance. Additionally, the emission spectrum of the donor chromophore has to overlap with the absorption (or excitation) spectrum of the acceptor. In order to realize single-molecule FRET detection the signal-to-noise ratio has to be sufficiently high, therefore the selection of the chromophores is crucial to obtain an excellent FRET pair. ${ }^{61-63}$ There are many commercially available fluorophores that can be combined and used for single-molecule spectroscopy based on FRET. In the case of CuAAC, AlexaFluor488 (AF488) alkyne and AlexaFluor594 (AF594) azide (Scheme 3) were used in order to map catalyst site location and catalytic reaction events (vide infra). Notice that this FRET pair has been chosen not only because of the emission spectrum of the donors overlaps very well with the absorption spectrum of the acceptor, but also because of the large separation between donor and acceptor emission profiles allows easy discrimination in favor of acceptor emission (Figure 1).

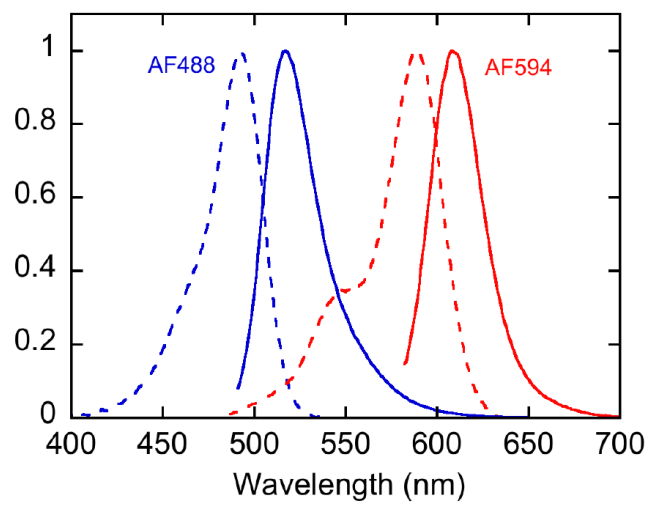

Figure 1. Normalized absorption (dashed) and emission (solid) spectra of AlexaFluor 488 alkyne and AlexaFluor 594 azide. Reprinted (adapted) with permission from J. Phys. Chem. Lett., 2015, 6 (20), pp 4049-4053. Copyright 2015. American Chemical Society.
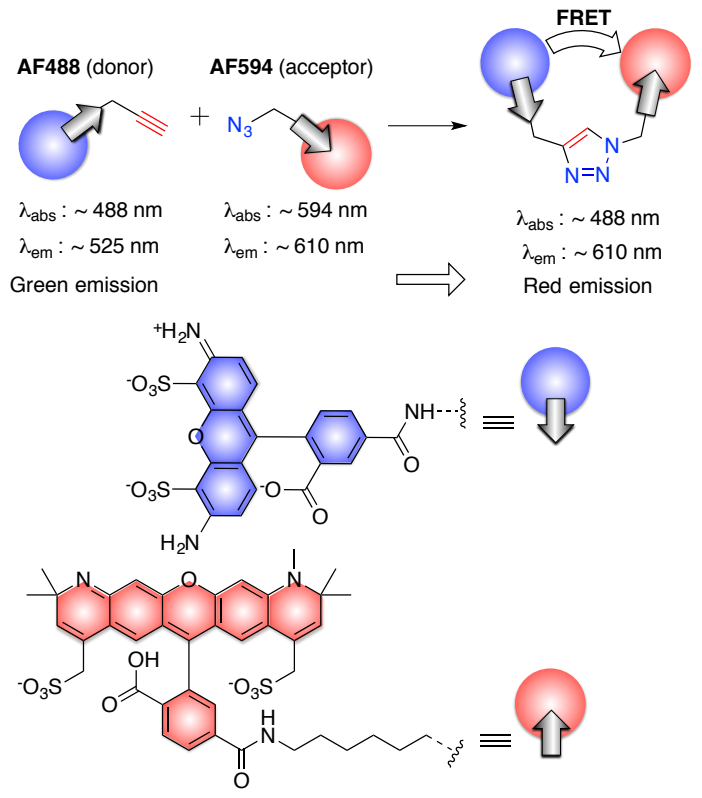

Scheme 3. Spectator dyes used in the single-molecule study of CuAAC.

\section{CATALYSIS AT THE SINGLE MOLECULE LEVEL}

The intimate knowledge on how a single molecule can reach a single-catalytic site, react and finally leave brings useful information not only on the catalytic mechanism but also in the catalyst site composition and distribution over the material. This knowledge cannot only give insights on the expected characteristics of the reactants and products, but also on the catalyst efficiency, helping in the design of more effective catalysts. In the subsections that follow, a couple of examples on our efforts to understand the catalytic efficiency of the $\mathrm{Cu}-$ AACs are shown as a proof of concept on how acquiring an intimate knowledge of the catalytic system can help to improve/re-design the catalyst effectiveness and bring this to the bench. First, we show how to use single-molecule spectroscopy (SMS) to determine whether or not the reaction occurs in the homogeneous or in the heterogeneous phase. ${ }^{53}$ Second, we focus on how single-molecule analysis can explore the catalytic efficiency and inspire new methodologies in order to improve or re-design catalytic materials. Finally, we emphasize the use of new photochemical strategies for CuAAC catalysis and comment on the potential of SMS techniques in organic chemistry.

\section{Heterogeneous catalysis}

As mentioned above, many attempts to determine the detailed nature of a catalytic mechanism fail at the bench scale approach. No matter how accurate the analytical technique used, the intimate interactions at the single-molecule/single-catalytic site level can only be revealed by spatio-temporal highresolution techniques that can follow the reaction under in situ and in real-time conditions. Single-molecule studies usually involve the use of concentrations in the picomolar-nanomolar range in order to make the effect of non-specific energy transfer statistically negligible and to separate catalytic sites beyond diffraction limit distances. Although, this can drastically interfere with the CuAAC reaction rates, the addition of an amine can assist by favoring the coordination of the reactants with the catalytic sites and avoiding possible formation of 
polynuclear unreactive copper species (as it typically happens at the homogeneous catalyzed $\mathrm{CuAAC}) .{ }^{64}$ Further, alkyne preassociation (see first equilibrium in Scheme 2) avoids the need of a three-way encounter between reagents and catalytic site, something that would be a major obstacle at the subnanomolar concentrations used.

When we first explored the CuAAC at the single-molecule level, colloidal copper nanoparticles were selected as the catalyst. ${ }^{53}$ The use of AF488 and AF596 helped to determine that the catalysis occurs at the heterogeneous phase, based on the premise that the absence of random solution events, and the presence of multiple bursting events in the same position reveal those catalytic spots where reaction events repeat themselves (see Chart 4). Therefore, the repetition of multiple events in the same location and the absence of random distributed events on the solution bulk are proof for heterogeneous processes..$^{53}$ Additionally, the duration of the ON times can be controlled by product desorption from the catalyst or due to product degradation, although in this case the latter possibility was ruled out by laser power dependence studies. ${ }^{53}$

One may ask: how does a successful catalytic event actually look as one is acquiring data in a microscope operating in TIRFM mode? Figure 2 illustrates representative data. In our case we normally capture a video consisting of 500 microscope frames over $50 \mathrm{~s}$. Different locations turn on and off as fluorescent bursts (indicative of reaction) take place. One of these frames, in panel A shows two bright locations that are 'on', one of them identified with an arrow. If we monitor this particular location we see temporal changes in intensity as illustrated in panel B where a fluorescence burst of approximately 12 seconds duration has occurred. A reaction occurred at about $13 \mathrm{~s}$ and the fluorescent molecule departed (and left the evanescent region) at about $25 \mathrm{~s}$ in the time axis. If one monitors this spot repeatedly or for a longer time one would see it turns on and off as catalytic events take place (Figure 3 ). Repeating bursts at the same location are evidence for heterogeneous catalysis. A 3D surface plot over the monitored area (typically $40 \times 40 \mu \mathrm{m}$ ) can be built by the addition of the intensity of each of the 500 images recorded, generating 3D map of reactivity as shown in panel $\mathrm{C}$. Note that all the catalytic spots (8 to 10 in panel $\mathrm{C}$ ) display comparable, but not identical intensity showing that every catalytic spot is slightly different, either in its activity or how long the product is retained.

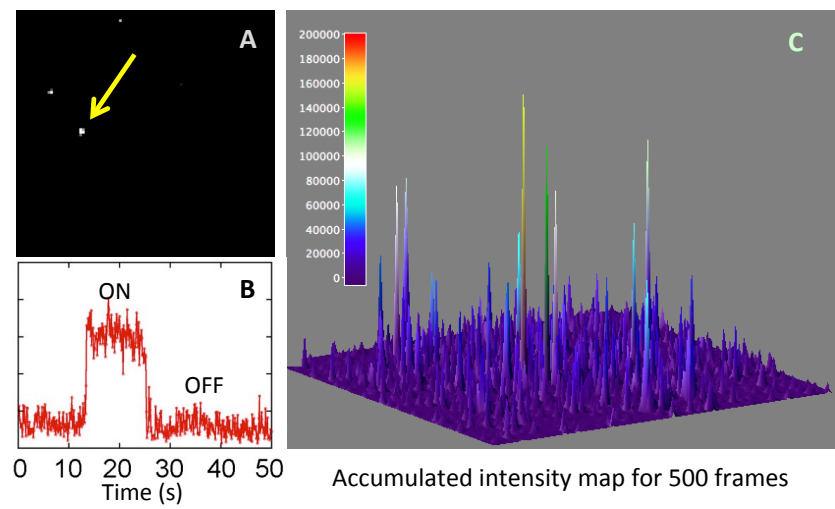

Figure 2. Selected data derived from TIRFM experiments using the dyes of Scheme 3 and with colloidal CuNP as catalyst. (A) Single frame from a 500 -frame video showing two locations where fluorescence bursts are active at the time of capture; (B) Intensity trajectory recorded at an active catalytic site, and (C) map of accumulated bursts over $50 \mathrm{~s}$ (500 frames) showing several sites where catalytic fluorescence bursts are clearly above the background signals.

Demonstrating that heterogeneous catalysis is occurring does not rule out the involvement of homogeneous reaction. As mentioned before, the formation of the $\mathrm{Cu}$-acetylide complex prior to the cycloaddition of the azide (Scheme 2) has a key role in the click reaction and here facilitates the localization of the reagent on the catalyst surface. Experiments ran exploring the same catalytic area show that the reactants interact with exactly the same surface area prior to reaction; thus the colocalization of the reactant and the product in the catalytic surface further suggests heterogeneous catalysis. Notice that due to the time limitation of the technique some homogeneous reactions can occur within the imaging timeframe and be undetectable. The limit of detection of homogeneous reactions using our system was determined $\leq 5 \%$ by flow experiments with the reaction product and in the absence of catalyst.

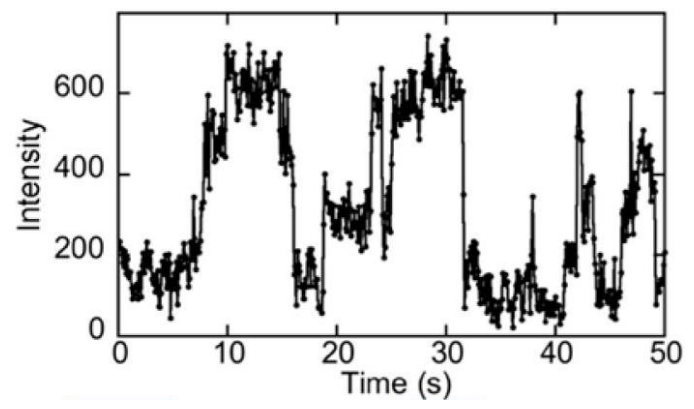

Figure 3. Multiple-events fluorescence trace found during TIRM experiment using dyes of Scheme 3 and with $\mathrm{Cu}$-on-charcoal as catalyst. $\mathrm{Re}$ printed (adapted) with permission from J. Phys. Chem. Lett., 2015, 6 (20), pp 4049-4053. Copyright 2015. American Chemical Society.

Finally, the time that a molecule (either reactant or product) spends in the catalyst surface and in solution is indicative of the type of interaction that this molecule has with the catalytic active site and can be used to explain pre-complexation or post-interaction process that can decrease the catalyst efficiency; the interplay of multiple factors affecting the catalytic outcome was nicely illustrated by Chen and coworkers for systems involving gold nanoparticles. ${ }^{25}$ In an earlier contribution exploring from the mole to the molecule strategies, ${ }^{65}$ we were able to measure the time the reactant and the product molecules spend in the catalyst surface using fluorescence correlation spectroscopy (FCS).

\section{Mapping the catalytic site}

Mapping the catalytic sites with sites that are far apart ( $>$ diffraction limit), can be easily performed following the average intensity of the burst events, Figure 2. Due to diffraction limitations, only super resolution (SR) microscopy can improve the resolution beyond this point. Several mathematical algorithms have been scripted in the past few years ${ }^{51}$ in order to accurately determine the central position of individual emitters point-spread function (PSF). The highly localized catalytic sites can give some insights on the efficiency of the catalyst. Our recent contribution in this field, helped us to establish the remarkably low "local" catalytic efficiency of $\mathrm{Cu}$-on-charcoal, usually regarded as an efficient catalyst. Although bench scale experiments show the catalyst can efficiently catalyze the $\mathrm{Cu}$ - 
$\mathrm{AAC},{ }^{39}$ our results show that the active surface of the catalyst is only $0.003 \%$, clearly leaving lots of room for improvement. The success of this study was based on the SR data analysis with sub-diffraction resolution $(\sim 20 \mathrm{~nm})$, see Figure 2 .

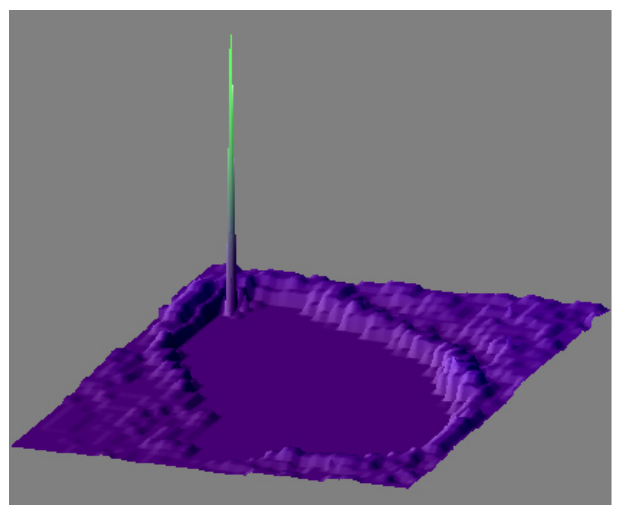

Figure 4. 3D map of reactivity of a piece of $\mathrm{Cu}$-on-charcoal catalyst from a reconstructed super-resolution image of the same region overlaying the white light image and the accumulated TIRF image $(4.2 \times 5.4 \mu \mathrm{m}$ area $)$. Reprinted (adapted) with permission from J. Phys. Chem. Lett., 2015, 6 (20), pp 4049-4053. Copyright 2015. American Chemical Society.

\section{From the molecule to the mole}

In organic synthesis, the performance of a catalyst is frequently measured in a rather quantitative way based on reaction time and equivalents of catalyst needed. In the field of catalysis the turnover number, or TON, is frequently used. TON is defined as the number of moles of product formed divided of the number of moles of catalyst. Rather more useful is the turnover frequency, TOF, defined as the number of molecules reacted per catalytic site and per unit time. The concepts are straightforward for homogeneous systems, where the numbers of catalytic sites equals the number of molecules (or moles) of catalyst. The situation is rather more complex for heterogeneous catalysis, where the number of catalytic sites is not easily obtainable in ensemble type experiments. Single molecule studies can directly observe the activity at a single catalytic site, as mentioned before for the case of $\mathrm{Cu}$-on-charcoal where a study of the large particles revealed that only one-in-ten particles had any catalytic activity and the particles were barely active. In the example shown in Figure 4, the catalytic site was less than $20 \mathrm{~nm}$ in size. Given the small fraction of the surface that is active, this seemed an ideal system to explore to what extent single molecule techniques could lead catalyst improvement. This low efficiency at the catalytic site level prompted us to the idea to examine the copper species anchored in the commercial $\mathrm{Cu}$-on-charcoal catalyst as they are not as reactive as anticipated. In our recent publication ${ }^{66}$ we proved the presence of high copper oxidation states in the commercial catalyst by X-ray Photon Spectroscopy (XPS) analysis and therefore we explored different strategies at the bench scale to improve its catalytic efficiency. Thus, reductive treatments of the material led to a dramatic increase in catalytic efficiency. The bench work was performed with a simple reaction (scheme 4) rather than the system of scheme 3 , as experiments with these substituted dyes would have a prohibitive cost at the scale required for bench work. Table 1 summarizes the results obtained at the bench scale when comparing the performance of the commercial catalyst (untreated) and the same catalyst treated in $\mathrm{EtOH}$ or with an aqueous solution of $\mathrm{NaBH}_{4}$.

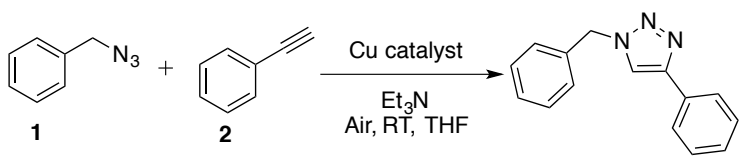

Scheme 4. CuAAC reaction used for bench scale experiments.

Table 1. Yields obtained for reaction in Scheme 4 using different concentration of catalysts after $3 \mathrm{~h}$ of reaction.

\begin{tabular}{|c|c|c|}
\hline \multirow{2}{*}{ Treatment } & \multicolumn{2}{|c|}{ Yield $(\%)^{\mathrm{a}}$} \\
\hline & 0.02 wt $\%$ & $0.2 \mathrm{wt} \%$ \\
\hline None & $<0.5(3.8)$ & $7.5(23.1)$ \\
\hline $2 \mathrm{~h}$ sonication in $\mathrm{EtOH}$ & $2.3(6.9)$ & $16.3(50.3)$ \\
\hline $2 \mathrm{~h}$ sonication in $\mathrm{NaBH}_{4}$ & $8.2(20.7)$ & $47.7(95.2)$ \\
\hline
\end{tabular}

a Values in brackets correspond to $12 \mathrm{~h}$ of reaction.

SMS on these systems would give more insights of the improvement at the catalytic site level. On closer examination we realized that the key manifestation of the catalytic improvement did not involve the large particles (as in Figure 4), but rather it occurred at other reproducible locations and in many cases with multiple fluorescence bursts, clear evidence of localized heterogeneous processes. These results could be readily rationalized with the presence of a second population of $\mathrm{Cu}$ on-charcoal particles with dimensions of $65 \pm 34 \mathrm{~nm}$ where the enhanced catalysis was centered. While these particles are not detectable by optical microscopy (i.e., smaller than the diffraction limit) the Scanning Electron Microscopy (SEM) imaging data and the single molecule fluorescence bursts reveal the origin of the microscopy data, fully consistent with the bench improvements. Particularly, the treatment with $\mathrm{NaBH}_{4}$ proved to increase the number of successful events per catalytic site. Although the improvement factor reached at the bench scale (10x or greater after treatment with $\mathrm{NaBH}_{4}$, Table 1 ) is clearly not the same at the single molecule level (Figure 5). These results are not surprising given that single molecule experiments are usually done in the $10^{-9}$ to $10^{-11} \mathrm{M}$ concentration range, while bench experiments were typically around 0.3 M. The possible requirement of two vicinal copper centers ${ }^{30}$ may also result in some differences between SMS and bench experiments. Additionally, the treatment with ethanol shows the same reactivity as the untreated material at the single molecule level, which account for the less drastic improvement ( $\sim 2 \mathrm{x}$ or less) found at the bench scale (Table 1). All these results are in agreement with the XPS analysis of the materials after treatment, which shows more reduced $\mathrm{Cu}$ species when the materials is subjected to $\mathrm{NaBH}_{4}$ treatment. Overall, we proved the SMS can be used not only to inspire improvements of the catalysis at the bench scale but also provides the tools to explain the nature of the improvement. 


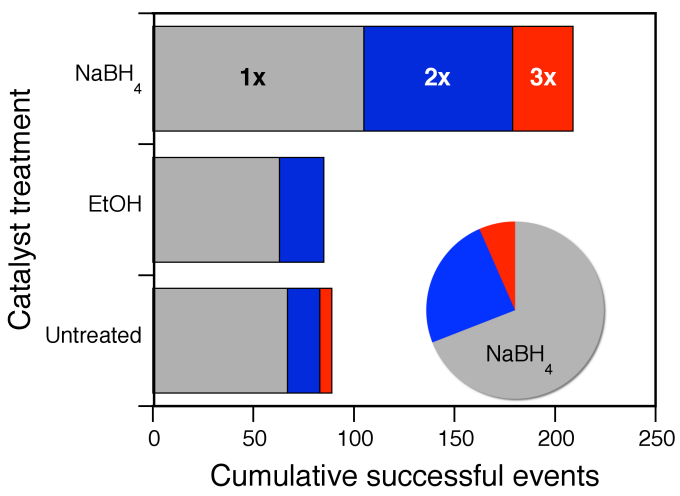

Figure 5: Cumulative successful events from analysis of an average of 4 videos per catalyst. Different colors correspond to intensity trajectories showing single event (grey), double events (blue) and triple events (red). The pie chart shows the distribution of traces with one, two or three events (same color pattern) found when the catalyst is pre-treated with $\mathrm{NaBH}_{4}$

\section{A photoactivated click catalyst inspired by les- sons learned with Copper-on-charcoal}

The studies mentioned above and the dramatic improvement of the catalytic activity of $\mathrm{Cu}$-on-charcoal particles achieved made it clear that the key to achieve good catalytic activity was to control carefully the oxidation state of copper, in particular the presence and abundance of $\mathrm{Cu}(\mathrm{I})$. With this in mind we wondered whether it would be possible to develop a light driven catalytic process ${ }^{67}$ where light could control the oxidation state of copper. Such a material would allow temporal and spatial control of click chemistry, which may be desirable for many applications, including as a tool for imaging techniques. The few examples on photo-activated click chemistry usually involve photoactivation of an organic reagent ${ }^{68}$ or use organic photoreducing agents to convert soluble $\mathrm{Cu}(\mathrm{II})$ to $\mathrm{Cu}(\mathrm{I})$, the active catalyst. ${ }^{69-71}$ Our approach can afford more flexibility as it relies on readily controllable photoactivation of the catalyst.

Having some experience with the use of $\mathrm{TiO}_{2}$ and $\mathrm{Nb}_{2} \mathrm{O}_{5}$ as semiconductors supports for photocatalysis, ${ }^{56,72-77}$ we tested the incorporation of copper nanoparticles on the surface of these nanostructures. Deposition of copper and exposure to ambient conditions leads to the formation of copper oxide $\left(\mathrm{CuO}_{\mathrm{x}}\right)$ nanoparticles, mainly constituted by $\mathrm{Cu}(\mathrm{II})$, rather than the required $\mathrm{Cu}(\mathrm{I})$. Thus, $\mathrm{CuO}_{\mathrm{x}} @ \mathrm{TiO}_{2}$ shows no catalytic activity in the dark, although the material based on niobium, $\mathrm{CuO}_{\mathrm{x}} @ \mathrm{Nb}_{2} \mathrm{O}_{5}$ shows some minor click catalysis, probably due to a few residual $\mathrm{Cu}(\mathrm{I})$ sites. Upon UVA excitation the materials become excellent photoactivated click catalysts. The catalytic activity is attributed to the UV excitation of a valence band $(\mathrm{VB})$ electron in $\mathrm{TiO}_{2}\left(\right.$ or $\left.\mathrm{Nb}_{2} \mathrm{O}_{5}\right)$ into the conduction band $(\mathrm{CB})$, a process that occurs readily in the UVA spectral region (320-400 nm), and is illustrated in Figure 6 for $\mathrm{CuO}_{\mathrm{x}} @ \mathrm{TiO}_{2}$. Both electron and hole, can be trapped by solvent or solutes to perform redox-type reactions in competition with rapid electron-hole recombination. The electron can also be scavenged by surface nanostructures, typically metal or metal oxides, a process that delays -but does not preventrecombination and enhances reaction opportunities.

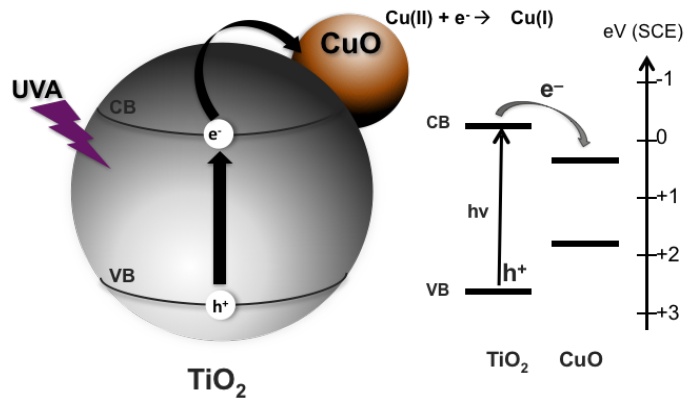

Figure 6: Proposed mechanism of electron transfer from the excited semiconductor to $\mathrm{CuO}$ nanoparticles to form catalytic $\mathrm{Cu}(\mathrm{I})$. Reprinted (adapted) with permission from J. Am. Chem. Soc., 2016, 138 (40), pp 13127-13130. Copyright 2016. American Chemical Society.

In the case of Figure 6, excitation of the $\mathrm{TiO}_{2}$ support effectively generates transient $\mathrm{Cu}(\mathrm{I})$ species, the desirable catalyst for $\mathrm{CuAAC}$ catalyst, as already noted for the $\mathrm{Cu}$-on-charcoal system. The materials proved to be excellent photocatalysts for the $\mathrm{CuAAC}$ reaction with good reusability. In the case of $\mathrm{CuO}_{\mathrm{x}} @ \mathrm{TiO}_{2}$ no decrease in activity was detected after four cycles, while for $\mathrm{CuO}_{\mathrm{x}} @ \mathrm{Nb}_{2} \mathrm{O}_{5}$ over $60 \%$ of the activity persisted after four cycles. This contribution is, to the best of our knowledge, the first example in which the $\mathrm{Cu}(\mathrm{I})$ species are photogenerated in situ. The reaction can be driven on demand simply by switching the light on and off; here the semiconductor is used as an electron shutter in order to generate the $\mathrm{Cu}(\mathrm{I})$ species needed for click chemistry.

The importance of controlling well the oxidation state of copper also becomes evident when one explores the effect of oxygen on the reaction. Figure 7 compares the kinetics of product formation under air and under argon, with plateau yields of about $32 \%$ and $92 \%$, respectively. However, if in the argon experiment the sample is opened to air after 8 hours the yield gradually 'recovers' to become comparable with that observed under air. This unusual effect is attributed to the overreduction of copper to inactive $\mathrm{Cu}(0)$. The oxidative cycle included in Figure 7 illustrates these concepts. We note that the copper nanoparticles do not need to be completely reduced to $\mathrm{Cu}(0)$ for their catalytic activity to stop; catalysis is a surface phenomenon and its probably sufficient for the surface to be reduced to $\mathrm{Cu}(0)$, with higher oxidation states likely present in the nanoparticle core.

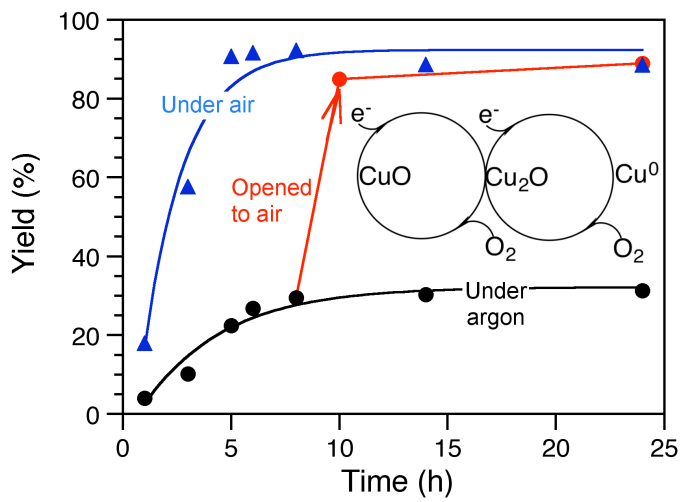

Figure 7. Study of the photocatalytic reaction of 1 and 2 (refer to Scheme 4) in THF in Ar (black) and in air (blue) using $\mathrm{CuO}_{\mathrm{x}} @ \mathrm{Nb}_{2} \mathrm{O}_{5}$. Red dots: yields obtained when system is exposed to air after $8 \mathrm{~h}$ of reaction under Ar. Inset: Role of the $\mathrm{O}_{2}$ in the $\mathrm{Cu}$ redox cycle. Reprinted (adapted) with 
permission from J. Am. Chem. Soc., 2016, 138 (40), pp 13127-13130. Copyright 2016. American Chemical Society.

Much to our surprise, these catalysts can be excited with either UV or visible light, which provide wavelength tuneability of the CuAAC that does not depend on the use of organic photoinitiators. While $\mathrm{TiO}_{2}$ does not absorb in the visible region, we have speculated that visible light activation involves absorption by small $\mathrm{CuO}$ nanoparticles that through an electron shuttle mechanism generate $\mathrm{Cu}(\mathrm{I})$ in larger nanoparticles in the $\mathrm{TiO}_{2}$ surface. ${ }^{26}$ The implication of such a mechanism is that nanoparticle polydispersity can aid the catalytic process. The intimate knowledge of the mechanism is still under consideration and relies on the use of single molecule spectroscopy.

\section{CONCLUSIONS}

Our title asks: Is single molecule fluorescence spectroscopy ready to join the organic chemistry toolkit? We believe the answer to this question is YES. As organic chemistry goes, SMS may still be for the adventurous, as we are at the dawn of a new approach for drug discovery, however, the observation of single molecules reacting at single catalytic sites, and the opportunity to map in detail the activity at catalytic structures opens new avenues for research in organic chemistry. These new avenues contrast with high throughput approaches largely based on chance; even discovery based on trial-and-error could use a tool that looks at the intimate details of the catalytic process as this understanding can lead to progress in reaction discovery and inspire the invention of improved catalysts.

While this contribution centers on click chemistry, other researchers -cited throughout this paper- have shown the applicability of SMS to several other examples. Single molecule techniques have developed to the point that they are accessible to non-experts, as long as they can afford turn-key instrumentation, expensive, but readily available. Studies using SMS can translate into major improvements at the bench as illustrated here for click chemistry.

\section{AUTHOR INFORMATION}

\section{Corresponding Author}

* Corresponding author's e-mail: titoscaiano@mac.com

\section{Author Contributions}

Both authors contributed equally to the preparation of this Perspective.

\section{Author Biographies}

Juan C. (Tito) Scaiano came to the National Research Council (NRG) in Ottawa from Argentina in 1975 to join the Ingold group. After holding a position at the University of Notre Dame (1976-1979), he returned to the NRC in 1979, where he studied organic reaction intermediates using laser techniques. In 1991, Scaiano joined the University of Ottawa where he is currently a Distinguished University Professor and holds the Canada Research Chair in Applied Photochemistry. Current interests include the study of organic photochemistry and reaction mechanisms, the application of nanomaterials to organic chemistry, single-molecule catalysis, and biomaterials. Scaiano is the 2016 winner of the ACS James Flack Norris Award in Physical Organic Chemistry.

Anabel E. Lanterna obtained her B.Sc. (2007) and Ph.D. (2013) degrees in Chemistry from National University of Córdoba in Argentina. She was a postdoctoral fellow for a short time in Rhodes University in South Africa under Prof. R. Krause supervision. She joined the Scaiano group at the University of Ottawa at the end of 2013 where she started her work in heterogeneous photoredox catalysis. Currently, she is interested in the design and study of new nanomaterials for their use in catalysis and health applications.

\section{Notes}

The present contribution is the result of an invitation in connection with the ACS James Flack Norris Award in Physical Organic Chemistry presented to J.C.S. at the 2016 Spring ACS meeting.

\section{ORCID Information}

Lanterna, A.E. - 0000-0002-6743-0940

Scaiano, J.C. - 0000-0002-4838-7123

\section{ACKNOWLEDGMENT}

We thank the Natural Sciences and Engineering Research Council of Canada, the Canada Research Chairs program and the Canada Foundation for Innovation for their generous support.

\section{ABBREVIATIONS}

CuAAC: copper(I)-catalyzed cyclization between azides and terminal alkynes; SMS: single molecule spectroscopy; TIRFM: Total Internal Reflection Fluorescence Microscopy; SR: Super Resolution; SEM: Scanning Electron Microscopy; XPS: X-ray Photon Spectroscopy.

\section{REFERENCES}

(1) The 2014 Nobel Prize in Chemistry was awarded to Eric Betzig, Stefan W. Hell and William E. Moerner for the development of super-resolved fluorescence microscopy

(2) Johnston, L. J. Langmuir 2007, 23, 5886.

(3) Godin, R.; Liu, H. W.; Smith, L.; Cosa, G. Langmuir 2014, 30,11138

(4) Esfandiari, N. M.; Wang, Y.; Bass, J. Y.; Cornell, T. P.; Otte, D. A. L.; Cheng, M. H.; Hemminger, J. C.; McIntire, T. M.; Mandelshtam, V. A.; Esfandiari, S. A. B. N. M.; Blum, S. A. J. Am. Chem. Soc. 2010, 132, 15167

(5) Esfandiari, N. M.; Blum, S. A. J. Am. Chem. Soc. 2011, 133, 18145.

(6) Cordes, T.; Blum, S. A. Nat. Chem. 2013, 5, 993.

(7) Blum, S. A. PCCP 2014, 16, 16333.

(8) Janssen, K. P. F.; De Cremer, G.; Neely, R. K.; Kubarev, A. V.; Van Loon, J.; Martens, J. A.; De Vos, D. E.; Roeffaers, M. B. J.; Hofkens, J. Chem. Soc. Rev. 2014, 43, 990.

(9) Sambur, J. B.; Chen, T. Y.; Choudhary, E.; Chen, G. Q.; Nissen, E. J.; Thomas, E. M.; Zou, N. M.; Chen, P. Nature 2016, 530,77 .

(10) Zhou, X. C.; Choudhary, E.; Andoy, N. M.; Zou, N. M.; Chen, P. ACS Catal. 2013, 3, 1448.

(11) Andoy, N. M.; Zhou, X. C.; Choudhary, E.; Shen, H.; Liu, G. K.; Chen, P. J. Am. Chem. Soc. 2013, 135, 1845.

(12) Chen, P.; Xu, W. L.; Zhou, X. C.; Panda, D.; Kalininskiy, A. Chem. Phys. Lett. 2009, 470, 151.

(13) Andoy, N. M.; Zhou, X.; Choudhary, E.; Shen, H.; Liu, G.; Chen, P. J. Am. Chem. Soc. 2013, 135, 1845.

(14) Ristanovic, Z.; Kubarev, A. V.; Hofkens, J.; Roeffaers, M. B. J.; Weckhuysen, B. M. J. Am. Chem. Soc. 2016, 138, 13586.

(15) De Cremer, G.; Sels, B. F.; De Vos, D. E.; Hofkens, J.; Roeffaers, M. B. J. Chem. Soc. Rev. 2010, 39, 4703.

(16) De Cremer, G.; Roeffaers, M. B. J.; Bartholomeeusen, E.; Lin, K.; Dedecker, P.; Pescarmona, P. P.; Jacobs, P. A.; De Vos, D. E.; Hofkens, J.; Sels, B. F. Angew. Chem. Int. Ed. 2010, 49, 908.

(17) Kubarev, A. V.; Janssen, K. P.; Roeffaers, M. B. ChemCatChem 2015, 7, 3646.

(18) Zheng, Z. K.; Tachikawa, T.; Majima, T. J. Am. Chem. Soc. 2014, 136, 6870. 
(19) Ng, J. D.; Upadhyay, S. P.; Marquard, A. N.; Lupo, K. M.; Hinton, D. A.; Padilla, N. A.; Bates, D. M.; Goldsmith, R. H. J. Am Chem. Soc. 2016, 138, 3876

(20) Rybina, A.; Lang, C.; Wirtz, M.; Grussmayer, K.; Kurz, A.; Maier, F.; Schmitt, A.; Trapp, O.; Jung, G.; Herten, D. P. Angew. Chem. Int. Ed. 2013, 52, 6322

(21) Weckhuysen, B. M. Angew. Chem. Int. Ed. 2009, 48, 4910

(22) Decan, M. R.; Scaiano, J. C. J. Phys. Chem. Lett. 2015 6,4049

(23) Macarron, R.; Banks, M. N.; Bojanic, D.; Burns, D. J.; Cirovic, D. A.; Garyantes, T.; Green, D. V. S.; Hertzberg, R. P.; Janzen, W. P.; Paslay, J. W.; Schopfer, U.; Sittampalam, G. S. Nat. Rev. Drug Discovery 2011, 10, 188.

(24) Kubarev, A. V.; Janssen, K. P. F.; Roeffaers, M. B. J. ChemCatChem 2015, 7, 3646.

(25) Xu, W.; Kong, J. S.; Yeh, Y.-T. E.; Chen, P. Nat. Mat. 2008, 7, 992.

(26) Wang, B.; Durantini, J.; Nie, J.; Lanterna, A. E.; Scaiano J. C. J. Am. Chem. Soc. 2016, 138, 13127.

(27) Kolb, H. C.; Finn, M. G.; Sharpless, K. B. Angew. Chem. Int. Ed. 2001, 40, 2004

(28) Moses, J. E.; Moorhouse, A. D. Chem. Soc. Rev. 2007, $36,1249$.

(29) Hein, J. E.; Fokin, V. V. Chem. Soc. Rev. 2010, 39, 1302

(30) Worrell, B. T.; Malik, J. A.; Fokin, V. V. Science 2013 $340,457$.

(31) Rodionov, V. O.; Fokin, V. V.; Finn, M. G. Angew. Chem. Int. Ed. 2005, 44, 2210

(32) Meldal, M.; Tornoe, C. W. Chem. Rev. 2008, 108, 2952.

(33) Alonso, F.; Moglie, Y.; Radivoy, G. Acc. Chem. Res. 2015, 48, 2516.

(34) Chassaing, S.; Sido, A. S. S.; Alix, A.; Kumarraja, M.; Pale, P.; Sommer, J. Chem. Eur. J. 2008, 14, 6713.

(35) Chassaing, S.; Beneteau, V.; Pale, P. Cat. Sci. Technol. 2016, 6, 923

(36) Alonso, F.; Moglie, Y.; Radivoy, G.; Yus, M. Eur. J. Org. Chem. 2010, 5913.

(37) Alonso, F.; Moglie, Y.; Radivoy, G.; Yus, M. Tetrahedron Lett. 2009, 50, 2358.

(38) Alonso, F.; Moglie, Y.; Radivoy, G.; Yus, M. Adv. Synth. Catal. 2010, 352, 3208.

(39) Lipshutz, B. H.; Taft, B. R. Angew. Chem. Int. Ed. 2006 45, 8235.

(40) Raut, D.; Wankhede, K.; Vaidya, V.; Bhilare, S.; Darwatkar, N.; Deorukhkar, A.; Trivedi, G.; Salunkhe, M. Catal. Commun. 2009, 10, 1240.

(41) Hudson, R.; Li, C. J.; Moores, A. Green Chemistry 2012 14,622

(42) Sharghi, H.; Khalifeh, R.; Doroodmand, M. M. Adv. Synth. Catal. 2009, 351, 207.

(43) Esfandiari, N. M.; Blum, S. A. J. Am .Chem. Soc. 2011, $133,18145$.

(44) Phan, N. T. S.; Van Der Sluys, M.; Jones, C. W. Adv. Synth. Catal. 2006, 348, 609 .

(45) Davies, I. W.; Matty, L.; Hughes, D. L.; Reider, P. J. J. Am. Chem. Soc. 2001, 123, 10139.

(46) McTiernan, C. D.; Pitre, S. P.; Scaiano, J. C. ACS Catal. 2014, 4, 4034 .

(47) Higman, C. S.; Lanterna, A. E.; Marin, M. L.; Scaiano, J. C.; Fogg, D. E. ChemCatChem 2016, 8, 2446.

(48) Pacioni, N. L.; Filippenko, V.; Presseau, N.; Scaiano, J. C. Dalton Trans. 2013, 42, 5832.
(49) Lipshutz, B. H.; Tasler, S.; Chrisman, W.; Spliethoff, B.; Tesche, B. J. Org. Chem. 2003, 68, 1177.

(50) Hodgson, G. K.; Impellizzeri, S.; Scaiano, J. C. Chem. Sci. 2016, 7, 1314

(51) Chen, P.; Zhou, X.; Shen, H.; Andoy, N. M.; Choudhary, E.; Han, K.-S.; Liu, G.; Meng, W. Chem. Soc. Rev. 2010, 39, 4560.

(52) Tao, F.; Crozier, P. A. Chem. Rev. 2016, 116, 3487.

(53) Decan, M. R.; Impellizzeri, S.; Marin, M. L.; Scaiano, J. C. Nat. Commun. 2014, 5, 4612.

(54) Wazawa, T.; Ueda, M. In Adv Biochem Eng Biot; Springer-Verlag Berlin Heidelberg: 2005; Vol. 95, p 77.

(55) Xu, W. L.; Kong, J. S.; Yeh, Y. T. E.; Chen, P. Nat. Mat. 2008, 7, 992

(56) Marin, M. L.; Hallett-Tapley, G. L.; Impellizzeri, S.; Fasciani, C.; Simoncelli, S.; Netto-Ferreira, J. C.; Scaiano, J. C. Catal. Sci. Tech. 2014, 4, 3044.

(57) Invitrogen ${ }^{\mathrm{TM}}$ Molecular Probes ${ }^{\mathrm{TM}}$ offers a wide variety of fluorescence reagents.

(58) Zheng, Q. S.; Juette, M. F.; Jockusch, S.; Wasserman M. R.; Zhou, Z.; Altman, R. B.; Blanchard, S. C. Chem. Soc. Rev. 2014, 43, 1044.

(59) Lakowicz, J. R. Principles of Fluorescence Spectroscopy; Springer US, 2006; Vol. 3.

(60) Turro, N. J. R., V.; Scaiano, J. C. Modern Molecular Photochemistry of Organic Molecules; University Science Publishers: New York, N.Y., 2010.

(61) Roy, R.; Hohng, S.; Ha, T. Nat Meth 2008, 5, 507.

(62) Jares-Erijman, E.; Jovin, T. Nat. Biotechnol. 2003, 21 1387.

(63) Hohlbein, J.; Craggs, T.; Cordes, T. Chem. Soc. Rev. 2014, 43, 1156

(64) Hein, J.; Fokin, V. Chem. Soc. Rev. 2010, 39, 1302.

(65) Carrillo, A. I.; Stamplecoskie, K. G.; Marin, M. L.; Scaiano, J. C. Cat. Sci. Technol. 2014, 4, 1989.

(66) B. Wang, J. D., M. R. Decan, J. Nie, A. E. Lanterna and J. C. Scaiano Chem. Commun. 2016, DOI: 10.1039/C6CC08905D.

(67) Green Chemistry; Luque, R., Ed.; Nova Science Publishers Inc: New York, United States, 2012.

(68) Arumugam, S.; Orski, S. V.; Mbua, N. E.; McNitt, C.; Boons, G. J.; Locklin, J.; Popik, V. V. Pure Appl. Chem. 2013, 85 1499.

(69) Tasdelen, M. A.; Yilmaz, G.; Iskin, B.; Yagci, Y. Macromolecules 2012, 45, 56

(70) Tasdelen, M. A.; Yagci, Y. Angew. Chem. Int. Ed. 2013, 52,5930 .

(71) Adzima, B.; Tao, Y.; Kloxin, C.; Deforest, C.; Anseth, K.; Bowman, C. Nature Chem. 2011, 3, 258.

(72) Morsella, M.; d'Alessandro, N.; Lanterna, A. E.; Scaiano, J. C. ACS Omega 2016, 1, 464.

(73) Lanterna, A. E.; Elhage, A.; Scaiano, J. C. Cat. Sci. Technol. 2015, 5, 4336.

(74) McTiernan, C. D.; Pitre, S. P.; Ismaili, H.; Scaiano, J. C. Adv. Synth. Catal. 2014, 356, 2819.

(75) Hallett-Tapley, G. L.; Crites, C.-O. L.; González-Béjar, M.; McGilvray, K. L.; Netto-Ferreira, J. C.; Scaiano, J. C. Journal of Photochemistry and Photobiology A: Chemistry 2011, 224, 8.

(76) Elhage, A.; Lanterna, A. E.; Scaiano, J. C. ACS Catal. 2016, 250.

(77) Impellizzeri, S.; Simoncelli, S.; Fasciani, C.; Luisa Marin, M.; Hallett-Tapley, G. L.; Hodgson, G. K.; Scaiano, J. C. Cat. Sci. \& Tech. 2015, 5, 169. 
Table of Contents artwork

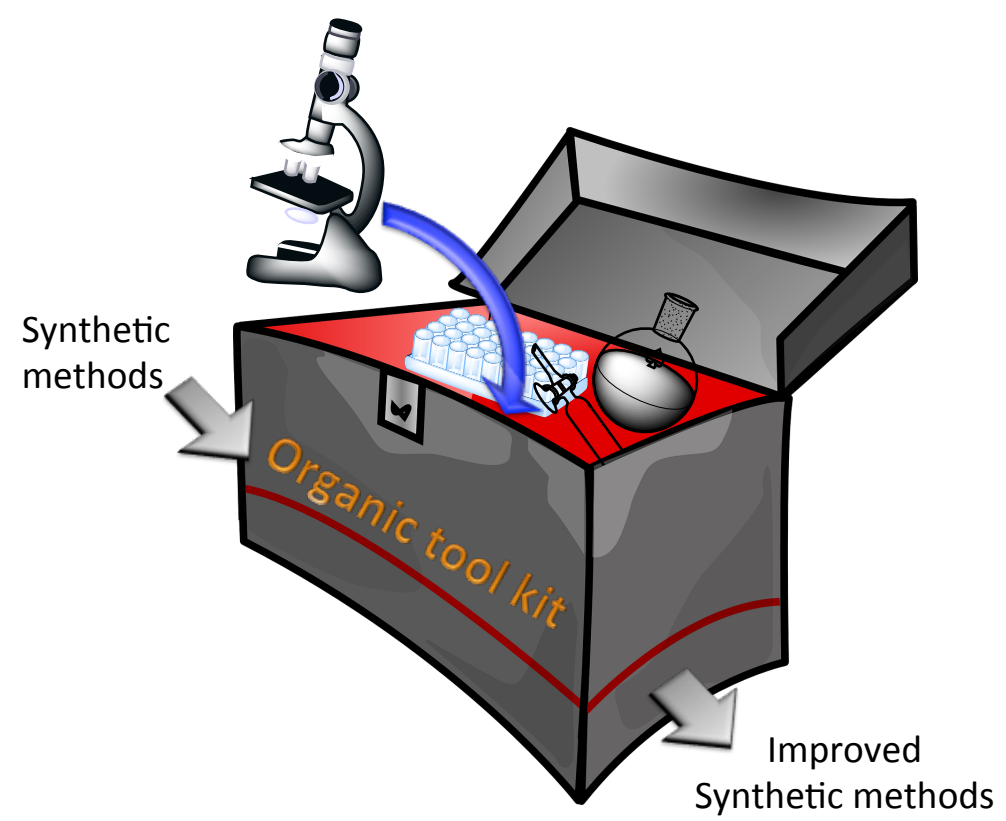


